\title{
AN EVALUATION OF THE SINGLE INJECTION THIOSULFATE METHOD FOR THE MEASUREMENT OF EXTRACELLULAR WATER ${ }^{1}$
}

\author{
By LEON C. CHESLEY AND ABRAHAM LENOBEL
}

\author{
(From the Department of Obstetrics and Gymecology, State University of New York College \\ of Medicine at New York City, and the Kings County Hospital, \\ Brooklyn, N. Y.)
}

(Submitted for publication July 19, 1956; accepted November 7, 1956)

The purpose of this paper is to examine the validity of the single injection thiosulfate method, proposed by Cardozo and Edelman (1) for the measurement of the extracellular fluid volume. The conclusion is that the method does not give a valid estimate of the extracellular fluid volume and probably does not even measure the volume of distribution potentially available to thiosulfate.

Cardozo and Edelman gave an accurately known amount of sodium thiosulfate (about $10 \mathrm{gm}$. of $\mathrm{Na}_{2} \mathrm{~S}_{2} \mathrm{O}_{3} \cdot 5 \mathrm{H}_{2} \mathrm{O}$ in 10 per cent solution) by intravenous injection over a period that varied from 7 to 16 minutes. They then collected venous blood samples at intervals and plotted the logarithms of the serum thiosulfate concentrations against time. The points usually fell on a straight line; this was extrapolated back to zero time, which arbitrarily was set as the moment the infusion had been started. If the points did not fall on a straight line, the test was discarded because of the variable clearance rate of the thiosulfate. The extrapolated value for serum concentration of thiosulfate $\left(P_{0}\right)$ is presumed to be what the concentration would be if the thiosulfate were instantaneously injected and evenly distributed in its final volume of dilution. This extrapolated $P_{0}$ is then divided into the total amount of thiosulfate injected to calculate the volume of distribution.

Aside from the fact that virtually none of the thiosulfate has been injected at this zero time, the method is based upon rather questionable assumptions, which will be discussed later.

In some of the evaluations of the single injection method that follow, comparisons were made between the volumes of distribution of thiosulfate and sucrose as calculated by different methods.

1 This investigation was supported by a research grant, H-1837 (C) from the National Institutes of Health, Public Health Service.
The other procedures used were the infusion/ slope method devised by Schwartz (2) and the calibrated infusion (IV minus UV) method of Deane, Schreiner, and Robertson (3).

\section{MATERIAL AND METHODS}

All experiments were done with hospitalized women. While they were not "normal," they were selected. Patients with any discernible cause for disturbance in hydration, such as fever, renal disease or heart disease, were excluded. All patients were less than age 50 years and most were in the third and fourth decades of life. Nearly all patients were fasting the day of the test. The few exceptions are those patients in whom simultaneous measurements were made of the volumes of distribution of sucrose and thiosulfate.

Reagent grade sodium thiosulfate $\left(\mathrm{Na}_{2} \mathrm{~S}_{2} \mathrm{O}_{3}\right)$ was made up as a 6.7 per cent solution in either isotonic saline or 5 per cent dextrose. This gives the same thiosulfate concentration as Cardozo and Edelman used in their 10 per cent solutions of the hydrated salt. The solution was sterilized by Seitz filtration and given intravenously from a calibrated buret. The dose was roughly $0.5 \mathrm{ml}$. per pound of body weight and was given over a period of from 8 to 14 minutes. Collections of venous blood were begun 25 minutes after the end of the infusion and five samples were taken over the next 75 minutes. When the volume of distribution was measured by the infusion/slope method, the priming dose was followed by constant infusion of more of the same solution at the rate of 1.05 to $1.10 \mathrm{ml}$. per minute for periods of 2 to 3 hours. The constant infusion was given by a Bowman pump, which was recalibrated after each use.

The sucrose solutions, used in measuring the volume of sucrose distribution, were made up in 8 per cent concentration in either isotonic saline or 5 per cent dextrose. (The latter gives an appreciable blank in the chemical method for measuring sucrose).

In the calibrated infusion method this solution was given intravenously at the rate of 1.05 to $1.10 \mathrm{ml}$. per minute. Half-hourly collections of venous blood and of urine were begun 2 hours after the start of the infusion. After the collection of 3 or $\mathbf{4}$ blood and urine samples the thiosulfate was given for the single injection procedure.

In measuring the volume of sucrose distribution by the 
infusion/slope method, a priming dose of 8 per cent sucrose was followed by more of the same solution, as described for the administration of thiosulfate. Usually, simultaneous measurements were made of the volumes of sucrose and thiosulfate distribution, with sucrose and thiosulfate combined in the same solution. In the infusion/slope procedure, a minimum of three venous blood samples were taken during the constant infusion, the first being obtained about 90 minutes after the beginning of the infusion. Five more venous blood samples were collected over the 100-minute period following the end of the infusion. In both the single injection method and the infusion/slope method the test was discarded in any case where the logarithms of the serum thiosulfate or sucrose concentrations, plotted against time, were not closely fitted by a straight line. Also no calculations were made from the data obtained by the infusion/slope method unless the serum concentrations of thiosulfate and sucrose had attained constancy.

The solutions injected were hypertonic, but the volumes were small in comparison to the volumes of distribution, being about 1 per cent in the single injection procedure. In the constant infusion methods the rate of administration was $1.07 \mathrm{ml}$. per minute, so that in 3 hours the total volume given was of the order of 2 to 3 per cent of the volume of distribution. Meanwhile renal excretion and metabolism of the solutes (except for sodium chloride) kept pace with the infusion rate in the cases studied by the infusion/slope method. Furthermore, the procedure was the same in all experiments except that solutions were sometimes given in 0.9 per cent saline and sometimes in $\mathbf{5}$ per cent dextrose.

For chemical analyses, two samples of the infusion solution were diluted $(1: 1,000$ for thiosulfate and $1: 2,000$ for sucrose); urine samples were suitably diluted (usually $1: 500$ or $1: 1,000$ ) and serum samples were prepared by precipitating the proteins. For thiosulfate the serum was diluted 5 times in the protein precipitation by tungstic acid and for sucrose it was diluted 10 or 20 times in precipitating the proteins by zinc hydroxide. All samples were analyzed in duplicate or quadruplicate. Thiosulfate was measured by the iodine titration method of Brun (4) and sucrose by the resorcinol method of Roe, Epstein, and Goldstein (5), using a Beckman DU spectrophotometer. Blood, urine, water and reagent blanks were analyzed with all measurements.
When sucrose infusions were given in 5 per cent dextrose, an aliquot of the original dextrose solution was diluted $1: 2,000$, as was the infusion solution. These dextrose blanks were carried through the sucrose analyses and their optical densities were substracted from the optical densities of the infusion samples. This correction factor averaged about 4 per cent of the readings of the infusion samples.

\section{RESULTS}

\section{Experimental alteration of the renal clearance rate}

The first experiments with the single injection method were designed to find what effect changing the slope of the exponential disappearance rate of serum thiosulfate would have upon $\mathrm{P}_{\mathrm{o}}$ and, therefore, upon the calculated volume of thiosulfate distribution. The slope of the exponential curve was changed in two ways: (a) by pre-medication with carinamide, which partially blocks the renal tubular excretion of thiosulfate (6), and (b) by intravenous theophylline ethylenediamine, which increases the glomerular filtration rate (7) and therefore the renal excretion rate of thiosulfate. More than half of the experiments could not be used because of the irregular effect of the drugs over the 100-minute period following the infusion of the thiosulfate. (This irregularity is reflected in a non-linear plot of the logarithms of the decreasing serum thiosulfate concentrations against time. Cardozo and Edelman wrote that such cases can not be used, for obvious reasons). By trial and error the best dose of carinamide was found to be $4 \mathrm{gm}$. given at 6:00 A.M. and $2 \mathrm{gm}$. given at 9:00 A.M., with the thiosulfate infusion given at 10:00 A.M. In the theophylline ethylenediamine experiments, $0.25 \mathrm{gm}$. of the drug was given intravenously about 10 minutes before the thiosulfate infusion and this was followed with a continuous infusion of another $0.25 \mathrm{gm}$. in 250

\section{TABLE I}

Experimental alteration of the clearance rate of serum thiosulfate by carinamide or theophylline, showing the times at which the experimental curves intersect with the curves found without medication and the percentage difference in calculated thiosulfate space resulting from the change in extrapolated $P$

\begin{tabular}{|c|c|c|c|c|c|c|c|c|c|c|}
\hline Experiment: & $1 a$ & $1 \mathrm{~b}$ & 2 & 3 & 4 & 5 & 6 & 7 & 8 & 9 \\
\hline $\begin{array}{l}\text { Minutes after zero time at which curves } \\
\text { cross: }\end{array}$ & 29 & 30 & 2 & No & 18 & 21 & 27 & 29 & 16 & 36 \\
\hline $\begin{array}{l}\text { Percentage difference in thiosulfate space } \\
\text { resulting from changed excretion rate: }\end{array}$ & 10 & 24 & 7 & 7 & 19 & 18 & 11 & 13 & 17 & 30 \\
\hline
\end{tabular}


ml. of 5 per cent dextrose over the 100 -minute period of the test.

Nine successful experiments were done in fasting women selected with special care as to "normality." In all cases the thiosulfate was given in 5 per cent dextrose. The order in which the experiments were done was varied from case to case and the tests were done on successive days in any one patient. All exponential disappearance curves for serum thiosulfate were fitted by the method of least squares to ensure objectivity. If the single injection method is valid, the disappearance curves should intersect at zero time, that is, $P_{o}$ should be constant because the amount of thiosulfate given was constant and the potential volume of distribution should not vary much from test to test (see Discussion).

Table I shows that the disappearance curves did not intersect at zero time; in one case the lines did not cross at all and in the majority of cases the intersections occurred at 20 to $30 \mathrm{~min}$ utes after zero time. The experimental alteration of the renal clearance rate of thiosulfate changed the calculated volume of distribution in every case, the change being from 7 to 30 per cent. One of the experiments is shown in Figure 1 , where the lines cross at 29 and 30 minutes. In this particular case, the thiosulfate space calculated from $P_{0}$ with carinamide is 138 per cent of that calculated from $\mathrm{P}_{0}$ with theophylline ethyl-

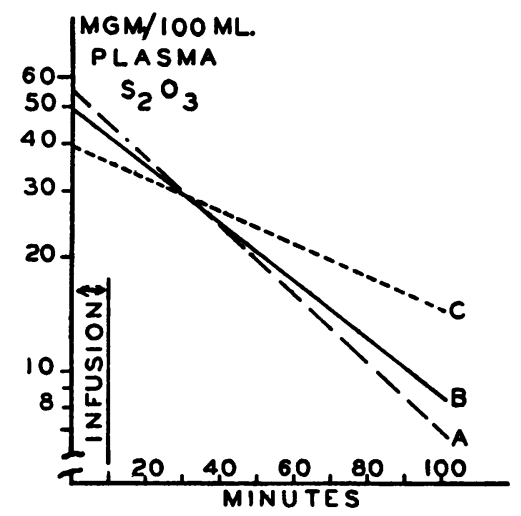

Fig. 1. The Effect of Altering the Renal Excretion of Thiosulfate Upon the Value of ExtraPOLATED $\mathrm{P}$ 。

$\mathrm{A}=$ pre-medication with theophylline ethylenediamine, $\mathrm{B}=$ basal measurement with no medication, $\mathrm{C}=$ premedication with carinamide. Note that $P_{0}$ is a function of the clearance rate of serum thiosulfate. enediamine; the calculated volumes were 11.7 and 8.5 liters, respectively. This is the only experiment, in more than 20 , in which three good disappearance curves were obtained. In the other eight cases reported there were two good curves, but the third with either carinamide or theophylline ethylenediamine departed from linearity on the semilog plot. Of the eight, five compare curves with no medication to curves with carinamide and three compare curves with no medication to those with theophylline ethylenediamine.

It would appear that the value for $\mathrm{P}_{\mathrm{o}}$ is a function of the clearance rate of thiosulfate. Experimental alteration of the renal clearance rate changes $P_{o}$ and results in changes in a predictable direction of the calculated volume of thiosulfate distribution. Decreasing the slope of the curve increases the calculated volume of distribution while increasing the slope of the curve decreases the calculated volume.

\section{Comparison of thiosulfate spaces measured by the infusion/slope method to those calculated from the single injection method}

The single injection method was compared with the infusion/slope method in six fasting patients, with alternation of the method first applied. In any one patient the tests were done on successive days. All solutions were given in 0.9 per cent saline.

The pairs of measurements did not check. In five of the six cases the volume of distribution for thiosulfate was considerably greater as measured by the infusion/slope method than as estimated from the single injection method. The results, in Table II, show that the average discrepancy was 30 per cent and ranged from 0 to 53 per cent.

Comparison of the thiosulfate space as estimated by the single injection method to the volume of sucrose distribution

In 18 patients the sucrose space was measured by the calibrated infusion method and immediately thereafter a single injection of sodium thiosulfate was given for the estimation of the volume of thiosulfate distribution. No experiment is included unless equilibrium of sucrose was established (that is, essentially constant volumes of 
TABLE II

Comparison of measurements of the thiosulfate space by the methods of Cardoso and Edelman and of Schwartz and of the slopes of the serum thiosulfate disappearance curves in the two methods

\begin{tabular}{|c|c|c|c|c|c|c|c|}
\hline Case: & 1 & 2 & 3 & 4 & 5 & 6 & Mean \\
\hline Body weight, $K g .:$ & 55.0 & 65.0 & 69.1 & 63.7 & 56.8 & 50.9 & \\
\hline $\begin{array}{l}\text { Thiosulfate space } \\
\text { Cardozo and Edelman: } \\
\text { Schwartz: }\end{array}$ & $\begin{array}{r}9.8 \\
12.0\end{array}$ & $\begin{array}{r}7.8 \\
11.9\end{array}$ & $\begin{array}{r}7.9 \\
11.5\end{array}$ & $\begin{array}{r}8.2 \\
11.5\end{array}$ & $\begin{array}{r}8.8 \\
11.6\end{array}$ & $\begin{array}{l}10.6 \\
10.6\end{array}$ & $\begin{array}{r}8.85 \\
11.50\end{array}$ \\
\hline $\begin{array}{l}\text { Ratio, Schwartz to Cardozo and } \\
\text { Edelman methods: }\end{array}$ & 1.22 & 1.53 & 1.46 & 1.40 & 1.32 & 1.00 & 1.30 \\
\hline $\begin{array}{l}\text { Ratio, disappearance curve slopes, } \\
\text { Cardozo and Edelman to Schwartz } \\
\text { methods: }\end{array}$ & 1.60 & 1.96 & 2.14 & 1.86 & 1.67 & 1.87 & 1.85 \\
\hline
\end{tabular}

distribution in successive periods). In all but two cases the solutions were given in 5 per cent dextrose. The calculations from the data for the single injection method gave apparent volumes of distribution for thiosulfate that averaged about 35 per cent less than the sucrose space, as also was found by Ikkos (8). The apparent volume of thiosulfate distribution, as calculated by the single injection method, bore no constant relationship to the volume of distribution of sucrose, as the scattering of points in Figure 2 shows. Of course, sucrose may not measure the extracellular fluid volume but under the conditions of the experiments a steady state had been attained and a reproducible space had been measured. (This often was not

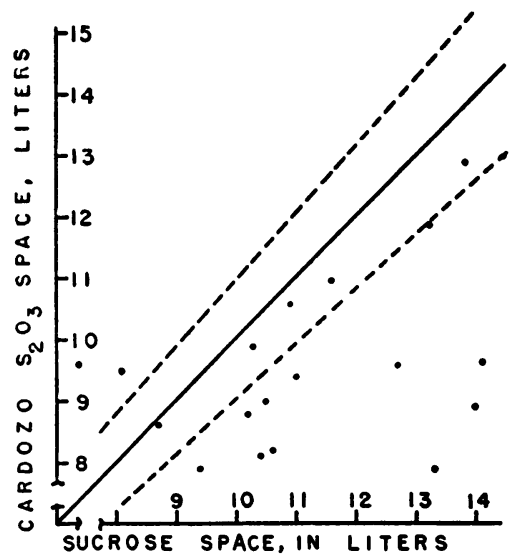

Fig. 2. Comparison of the Thiosulfate Space, as Calculated by the Cardozo and Edelman Single INjection Method, to the Sucrose Space Measured AlMost Simultaneously

The solid line is the line of identity and the broken lines represent plus and minus 10 per cent.

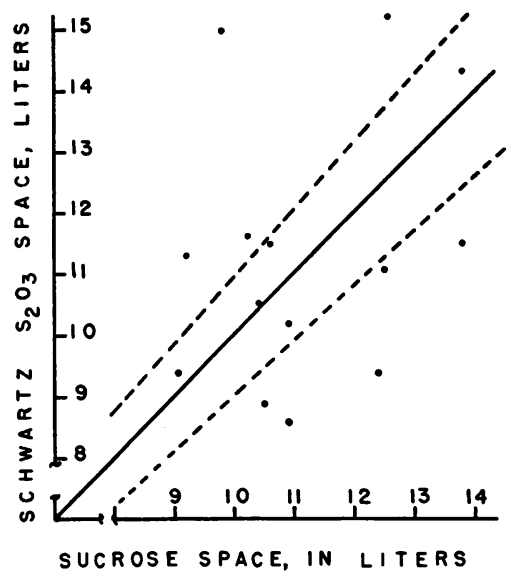

Fig. 3. Comparison of the Thiosulfate Space, as Measured by the Schwartz Infusion/Slope Method, to the Sucrose Space Measured Simultaneously

The solid line is the line of identity and the broken lines represent plus and minus 10 per cent.

true in Ikkos' experiments, for in about 80 per cent of his cases the apparent volume of sucrose distribution increased steadily with time. Arbitrarily, he took the apparent volume of distribution at 150 minutes for comparison with apparent volumes of thiosulfate distribution.)

Comparison of the thiosulfate space measured by the infusion/slope method with the volume of distribution of sucrose

Measurements of the volume of distribution of thiosulfate, made with the infusion/slope method, were compared with simultaneous measurements of the volume of sucrose distribution in 14 patients. In nine of these the volume of sucrose distribution 
TABLE III

Comparison of thiosulfate and sucrose spaces measured simultaneously by the infusion/slope method of Schwartz

\begin{tabular}{|c|c|c|c|c|c|c|c|c|c|c|c|c|c|c|}
\hline Case: & 1 & 2 & 3 & 4 & 5 & 6 & 7 & 8 & 9 & 10 & 11 & 12 & 13 & 14 \\
\hline Sucrose space, $l$. & 10.4 & 13.7 & 9.0 & 10.9 & 10.6 & 12.5 & 10.2 & 10.5 & 13.8 & 12.5 & 10.9 & 9.2 & 12.4 & 9.8 \\
\hline Thiosulfate space, $l$. & 10.5 & 14.3 & 9.4 & 10.2 & 11.5 & 11.1 & 11.6 & 8.9 & 11.5 & 15.1 & 8.6 & 11.3 & 9.4 & 15.1 \\
\hline $\begin{array}{l}\text { Percentage deviation } \\
\text { of thiosulfate space } \\
\text { from sucrose space }\end{array}$ & 1 & 4 & 5 & 6 & 8 & 11 & 14 & 15 & 16 & 21 & 21 & 23 & 24 & 54 \\
\hline
\end{tabular}

was measured both by the infusion/slope method and by the calibrated infusion method; in the other five, one or the other of the methods was used.

Figure 3 shows that there was no consistent relationship between the thiosulfate and sucrose volumes of distribution, although the average difference was very small. Relative to the volume of sucrose distribution, the thiosulfate space varied from plus 54 to minus 24 per cent, as shown in Table III. While the average ratio is close to 1.0, the wide scatter of the points in Figure 3 indicates that this may be fortuitous.

Comparison of volumes of sucrose distribution measured simultaneously by the infusion/slope and calibrated infusion methods

Inasmuch as the calculations of the volume of thiosulfate distribution did not agree when the

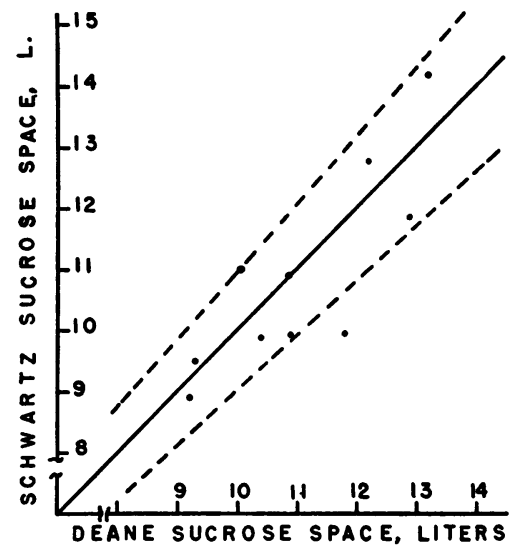

Fig. 4. Comparison of Simultaneous Measurements of the Sucrose Space Made by the Schwartz Infusion/Slope Method and by the Deane, Schreiner, and Robertson Calibrated Infusion (IV Minus UV) METHOD

The solid line represents identity and the broken lines plus and minus 10 per cent. single injection and the infusion/slope methods were applied successively in individual patients, it is of interest to compare the infusion/slope method to some other procedure. Therefore simultaneous measurements of the volume of sucrose distribution were made by the infusion/slope and the calibrated infusion methods. Here the sucrose was given in 0.9 per cent saline and the priming dose of sucrose was added in the "IV" factor of the IV minus UV formula of the calibrated infusion method.

As Figure 4 shows, these methods, quite different in principle, gave very similar volumes for the sucrose distribution. All but 1 of the 10 pairs of measurements checked within 10 per cent. The points shown in the graph are symmetrically distributed about the line of identity and the average difference between the simultaneous measurements is zero. Here, the average ratio of 1.0 may not be fortuitous for, in contrast to the comparison of volumes of thiosulfate and sucrose distribution, the pairs of measurements check closely.

\section{DISCUSSION}

The apparent advantage of the single injection method lies in its circumvention of the constant infusion and urine collections. However, its theoretic bases do not seem sound. Thiosulfate is known to enter the red blood cells (1) and if it enters these cells it may enter others. Gilman, Philips, and Koelle (9) found that in dogs about one-fourth of the injected thiosulfate cannot be recovered and they concluded, on good evidence, that most of the irretrievable fraction disappeared during and just after the infusion. The incomplete recovery was confirmed, for man, by Cardozo and Edelman. This together with the odor of the urine after thiosulfate injection suggest that 
thiosulfate is metabolized in the body. Inasmuch as it is relatively stable in blood, in vitro, such metabolism probably occurs in cells.

The extrapolation of the decremental curve back to zero time implies questionable assumptions. One assumption is that thiosulfate disappears at the same exponential rate from the beginning of the infusion to the end of the blood collections (100 minutes). The assumed starting point for this exponential decrement in plasma concentration is based not upon the amount already injected at zero time, but upon the total amount to be injected over the next 10 or more minutes. This does not seem reasonable. Also, even when such injections are given within seconds, the early portion of the decremental curve is not adequately described by the course of the exponential limb, as shown by Sheppard, Overman, Wilde, and Sangren (10) and by Sapirstein, Buckley, and Ogden (11). Another assumption is shown graphically in the first figure in the paper by Cardozo and Edelman. The early portion of the decremental curve lies above the extrapolated exponential limb (as in all such cases); Cardozo and Edelman factor this early portion of the curve into two straight (exponential) lines by the standard procedure of subtracting the extrapolated limb from the observed points. From this they draw the conclusion that two processes are going on in the early minutes : $(a)$ equilibration of the thiosulfate throughout plasma and interstitial fluid, and (b) clearance of thiosulfate at a uniform exponential rate, by renal excretion and metabolism. However, the rapid component of the decremental curve, in man, may represent the immediate loss of thiosulfate, which Gilman, Philips, and Koelle found in dogs. If the steep curve represents equilibration of thiosulfate throughout all of extracellular water, as Cardozo and Edelman suggest, the distribution must occur with almost incredible rapidity-within 10 minutes, as they say. In view of the time ( 30 to 120 minutes) required for sucrose to attain its maximum volume of distribution in normal subjects, it does not seem likely that thiosulfate would become evenly distributed in so short a time. The diffusion coefficients can not be specified exactly because of concentration effects. Cardozo and Edelman cited $0.68 \mathrm{~cm} .{ }^{2}$ per day for thiosulfate and Raisz, Young, and Stinson (12) cited $0.55 \mathrm{~cm}^{2}$ per day for su-

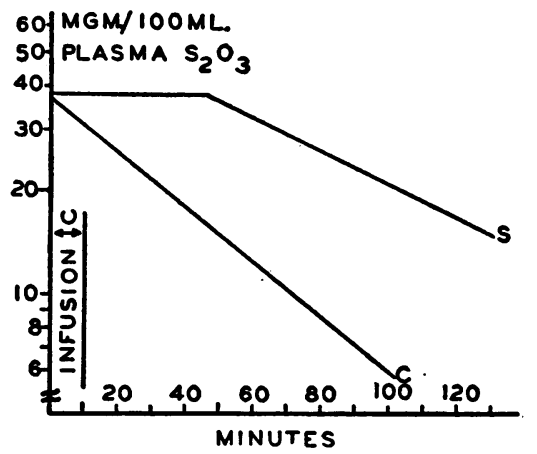

Fig. 5. Comparative Rates of Clearance of Serum Thiosulfate IN The Schwartz Infusion/Slope Method (S) and Cardozo and Edelman Single INJeCtion Method (C)

The constant infusion of the Schwartz method had gone on for 2 hours before the beginning of the time shown in the graph, allowing time for the establishment of a steady state. "Infusion C" refers to the period of infusion in the Cardozo and Edelman method.

crose; the source in both cases is the International Critical Tables.

One observation is consistent with the interpretation that the steep component may represent immediate loss of thiosulfate. Figure 5 and the last line in Table II show that the slope of the serum thiosulfate disappearance curve was nearly twice as great in the single injection procedure as in the infusion/slope method. This suggests that the prolonged infusion of the latter method may have "saturated" some space not so "saturated" in the much shorter period of the single injection procedure, where thiosulfate may still be leaving the blood stream by diffusion into extravascular fluid throughout the whole course of observation. Possibly this "saturation" occurring in the prolonged period of constant infusion represents the attainment of concentration equilibrium between plasma and extravascular fluid available to thiosulfate. There are other possible factors in the slower rate of clearance from the plasma, after prolonged infusion. One might be increased feedback from extravascular sources; this, again, would suggest that in the single injection method the extravascular sources had not been brought into equilibrium with plasma water. Another possibility is that the rate of metabolism of thiosulfate slows down after prolonged infusion.

If a large proportion of the injected thiosulfate does disappear quickly, in man, then obviously the 
dividend in the equation for the single injection method is too great and, of itself, should give calculated volumes of distribution that are too large. However, the volume calculated from this equation averages 30 per cent less than the measurements of the thiosulfate space by the infusion/ slope method. This suggests that the divisor $\left(\mathrm{P}_{\mathrm{o}}\right)$ in the equation for the single injection method is too large and in even greater error than is the dividend. This could result from the failure of thiosulfate to diffuse into and attain equilibrium in its potential total volume of distribution within the short period of time encompassed in the test. Thus, at least two variable and partially compensatory errors fortuitously result in calculations of an apparent thiosulfate space that often falls within the range described as normal for the volume of distribution of inulin.

The experimental alteration of the renal clearance of thiosulfate changes $\mathrm{P}_{o}$ and therefore the apparent volume of thiosulfate distribution as calculated in the single injection method. Since this work was completed, Peterson, O'Toole, and Kirkendall (13) have reported marked variations in the sucrose space of man, during a 24-hour period. Inasmuch as the present experiments, in which the renal clearance rate of thiosulfate was varied, were done on successive days, the assumption that the space should be nearly constant from day to day may be in error. In the three patients studied in regard to such reproducibility there was less than 5 per cent variation in the calculated volume of the thiosulfate distribution, from day to day. No more cases were studied because of the relative constancy reported for repeated estimations by Cardozo and Edelman (1), Ikkos (8), Raisz, Young, and Stinson (12) Becker and Joseph (14) and others. The reproducibility seems to be within the limits of analytic error. Even if it were not, changing the renal clearance rate of thiosulfate has invariably changed the calculated volume of distribution in a predictable direction.

\section{SUM MARY}

Experimental alteration of the renal excretion rate of thiosulfate, by pre-medication with carinamide or theophylline ethylenediamine, changed significantly the volume of thiosulfate distribution as calculated by the single injection method.
The volume of thiosulfate distribution, as measured by the infusion/slope method, averaged 30 per cent greater than the space calculated by the single injection method.

The thiosulfate space, measured by the infusion/slope method or calculated by the single injection method, bore no constant relation to the volume of sucrose distribution.

Simultaneous measurements of the sucrose space by the infusion/slope and by the calibrated infusion methods gave consistent results.

The failure of the single injection method to give thiosulfate spaces even approximating those measured by the infusion/slope method strongly suggests that the single injection method does not measure the volume of thiosulfate distribution.

It is concluded that the single injection method is not sound theoretically and that apparent volumes of distribution calculated from $\mathrm{P}_{0}$ have no significance as spaces. They only fortuitously come close to volumes measured by sucrose or inulin because of multiple, variable and partially compensatory errors.

\section{ACKNOWLEDGMENT}

Miss Pauline Scotto-Lavino assisted in many of the sucrose and thiosulfate analyses.

\section{REFERENCES}

1. Cardozo, R. H., and Edelman, I. S., The volume of distribution of sodium thiosulfate as a measure of the extracellular fluid space. J. Clin. Invest., 1952, 31, 280.

2. Schwartz, I. L., Measurement of extracellular fluid by means of a constant infusion technique without collection of urine. Am. J. Physiol., 1950, 160, 526.

3. Deane, N., Schreiner, G. E., and Robertson, J. S., The velocity of distribution of sucrose between plasma and interstitial fluid, with reference to the use of sucrose for the measurement of extracellular fluid in man. J. Clin. Invest., 1951, 30, 1463.

4. Brun, C., Thiosulfate determination in kidney-function tests. A simple method for the determination of thiosulfate in blood and urine. J. Lab. \& Clin. Med., 1950, 35, 152.

5. Roe, J. H., Epstein, J. H., and Goldstein, N. P., Photometric method for the determination of inulin in plasma and urine. J. Biol. Chem., 1949, 178, 839.

6. Lambiotte, C., Blanchard, J., and Graff, S., Thiosulphate clearance in pregnancy. J. Clin. Invest., 1950, 29, 1207. 
7. Chasis, H., Ranges, H. A., Goldring, W., and Smith, H. W., The control of renal blood flow and glomerular filtration in normal man. J. Clin. Invest., 1938, 17, 683.

8. Ikkos, D., Comparison of the volume of distribution of sucrose, inulin and thiosulfate in human subjects. Metabolism, 1955, 4, 19.

9. Gilman, A., Philips, F. S., and Koelle, E. S., The renal clearance of thiosulfate with observations on its volume distribution. Am. J. Physiol., 1946, $146,348$.

10. Sheppard, C. W., Overman, R. R., Wilde, W. S., and Sangren, W. C., Disappearance of $\mathrm{K}^{23}$ from nonuniformly mixed circulation pool in dogs. Circ. Research, 1953, 1, 284.
11. Sapirstein, L. A., Buckley, N. M., and Ogden, E., Rate of extravasation of intravenously injected thiocyanate in the dog. Am. J. Physiol., 1955, 183, 178.

12. Raisz, L. G., Young, M. K., Jr., and Stinson, I. T., Comparison of the volumes of distribution of inulin, sucrose and thiosulfate in normal and nephrectomized dogs. Am. J. Physiol., 1953, 174, 72.

13. Peterson, R. E., O’Toole, J. J., and Kirkendall, W. M., The variability of extracellular fluid space (sucrose) in man during a 24-hour period. J. Clin. Invest., 1956, 35, 730.

14. Becker, E. L., and Joseph, B. J., Measurement of extracellular fluid volumes in normal dogs. Am. J. Physiol., 1955, 183, 314. 